\title{
Gestión del Conocimiento para elevar niveles de desempeño laboral en las Pymes.
}

\author{
Knowledge Management to raise levels of job performance in \\ SMES.
}

\author{
Ing. Martha Cecilia Bravo Cedeño ${ }^{1}{ }^{*}$, Ing. Rita Jaritza Muñoz Salgado ${ }^{2}$ \\ 1.* Magister en Administración de Empresas Mención en Gerencia de la Calidad y Productividad, Universidad laica Eloy Alfaro \\ de Manabí Extensión Chone, Chone, Ecuador. Email: martha.bravo@uleam.edu.ec \\ ORCID: https://orcid.org/0000-0002-8403-7112
}

2. Magister en Administración de Empresas Mención en Gestión de Pequeñas y Medianas Empresas Agroproductivas, Universidad laica Eloy Alfaro de Manabí Extensión Chone, Chone, Ecuador. Email: rita.munoz@uleam.edu.ec

ORCID: https://orcid.org/0000-0001-9556-1414

\author{
Recibido: 06/ enero/2021 Aceptado: 10/febrero/2021 Publicado: 31/marzo/2021
}

Destinatario: martha.bravo@uleam.edu.ec

\begin{abstract}
Como citar: Bravo Cedeño, M. C., \& Muñoz Salgado, R. J. (2021). Gestión del Conocimiento para elevar niveles de desempeño laboral en las Pymes. Revista E-IDEA Journal of Business Sciences, 3(9), 1-12. https://doi.org/10.53734/eidea.vol3.id83
\end{abstract}

\begin{abstract}
Resumen: La sociedad del conocimiento propicia el surgimiento de una nueva civilización y de una nueva economía que se basa, no en los recursos materiales, sino en el "saber y el hacer saber" de las personas y de las organizaciones que, en desafíos inciertos de marcada complejidad, apuntan a fortalecer la construcción del conocimiento con el fin de lograr provecho de las oportunidades y mejorar los niveles de desempeño laboral en ellas. Esta realidad involucra a las microempresas o llamadas Pymes, las cuales en el ámbito del emprendimiento deben luchar para sobrevivir y permanecer en este; La investigación tiene como objeto, generar una aproximación teórica sobre la gestión del conocimiento para elevar los niveles de desempeño laboral en las Pymes, centrada en un enfoque cualitativo desde una perspectiva fenomenológica-hermenéutica. Para el logro de este objetivo, se diseñaron y aplicaron entrevistas semiestructuradas a los actores sociales (inversionistas- empleadosgerentes-supervisores), que fueron los informantes claves de este estudio, se utilizaron técnicas propias del método, para la categorización de la información, interpretación y compresión, logrando en efecto la reducción eidética, producto de la interpretación contextual de la información. Resaltando finalmente, la visión integradora de la gestión del conocimiento que permite lograr elevar los niveles de desempeño laboral en las Pymes del empleado, acorde a las actitudes y comportamientos del inversionista-gerente y supervisor a través del aprovechamiento y utilización del conocimiento del talento humano en las microempresas.
\end{abstract}

Palabras Clave: Gestión del conocimiento, desempeño laboral, microempresas o Pymes.

Abstract: The knowledge society fosters the emergence of a new civilization and a new economy that is based, not on material resources, but on the "knowing and making known" of people and organizations that, in uncertain challenges of marked complexity, aim to strengthen the construction of knowledge in order to take advantage of opportunities and improve job performance levels in them. This reality involves micro-enterprises or so-called SMEs, which in the field of entrepreneurship must fight to survive and remain in it; The research aims to generate a theoretical approach on knowledge management to raise the levels of job performance in SMEs, focused on a qualitative approach from a phenomenological- hermeneutical perspective. To achieve this objective, semi-structured interviews were designed and applied to the social actors (investors-employeesmanagers-supervisors), who were the key informants of this study, techniques of the method were used to categorize the information, interpretation and compression, achieving in effect the eidetic reduction, product of the contextual interpretation of the information. Finally, highlighting the integrative vision of knowledge management that allows raising the levels of job performance in SMEs of the employee, according to the attitudes and behaviors of the investor-manager and supervisor through the use and use of knowledge of human talent in micro-enterprises.

Keywords: Knowledge management, job performance, microenterprises or SMEs. 


\section{INTRODUCCIÓN}

$\mathrm{L}$ os fenómenos físicos, biológicos, psicológicos, sociales, políticos, económicos y ambientales representan cada vez más en este siglo XXI, escenarios complejos que se interconectan entre sí, lo que genera curiosidad en los diferentes campos del saber, obligando a desarrollar estudios en este ámbito. Esta realidad ha dado paso a una sociedad fundamentada cada vez más en el conocimiento, el cual se establece como el eje básico para el desarrollo y el progreso. En efecto, tanto las organizaciones y, por ende, las personas tienen como reto hacer frente a esta dinámica, que exige una clara distribución hacia la reproducción de nuevos conocimientos que permitan dar aportes característicos a las demandas de la sociedad o campo de trabajo donde se desenvuelve.

En este orden de ideas toma relevancia el concepto de la gestión del conocimiento el cual se ha ido transformando dinámicamente en los últimos años; naciendo en las teorías de la administración empresarial y, posteriormente, sigue como una idea más compleja, ligada a la emergencia de la sociedad del conocimiento y de la información.

Por su parte, lo complejo del entorno actual saturado de competencia, de problemas sociales, de salud, políticos, entre otros, conlleva a pensar en las organizaciones, darles sentido de dirección, rediseñar u optimizar los procesos esenciales, desarrollar estructuras organizacionales para que dichos procesos o planes funcionen pertinentemente, hacer uso adecuado de la tecnología en función del logro de los objetivos previstos para la organización.

Cabe destacar, que la gestión del conocimiento ha impactado a las técnicas gerenciales, manifestándose en las propuestas de nuevos enfoques metodológicos para abordar e interpretar la realidad a través de nuevos paradigmas, que tiene como fin valorizar y aprovechar la creación y la transmisión de conocimientos en todas las organizaciones.

En tal sentido, comenzó a resultar evidente que la gestión de las empresas como organizaciones y los niveles de desempeño laboral estaba ligado a la gestión del conocimiento. En efecto, cabe señalar que dentro de este contexto se encuentran las PYMES como organizaciones empresariales basadas en el conocimiento y cuya misión es aprovechar, generar o crear, transmitir y difundir conocimiento de sus productos y/o servicios.En el contexto de las PYMES del Ecuador, según estudios se ha determinado: que una de sus transcendentales debilidades es la falta de formalidad en las relaciones laborales con sus participantes lo que provoca una alta rotación del personal, el mismo que el momento que abandona la organización se lleva consigo un cúmulo de experiencias de aprendizaje personal que se conoce como conocimiento tácito, ocurriendo con ello que la empresa tenga que realizare invertir en nuevos costos de preparación, capacitación y desarrollo.

En consecuencia, la investigación consistió en generar una aproximación teórica de la gestión del conocimiento en el contexto de las PYMES para elevar los niveles de desempeño laboral. Seleccionando el enfoque para aproximar al fenómeno a conocer, en el contexto de

Bravo Cedeño, M. C., \& Muñoz Salgado, R. J. (2021). Gestión del Conocimiento para elevar niveles de desempeño laboral en las Pymes. E-IDEA Journal of Business Sciences, 3(9), 1-14. 
la investigación cualitativa de cada uno de los componentes de la gestión del conocimiento en las PYMES y de los significantes en los niveles de desempeño laboral que esta genera, a través de su análisis profundo, coherente y dinámico.

Para dar cumplimiento a este estudio, el artículo estará estructurado como sigue: en el primer apartado se describirá un poco la problemática del tema, seguido se harán definiciones claves sobre gestión del conocimiento, exponiendo los aspectos teóricos del conocimiento y las PYMES, y se realizará un enfoque en el fenómeno a estudiar y las estrategias para interpretar la realidad. Por último, se presentarán las conclusiones más relevantes que soportarán los aportes trascendentales del fenómeno estudiado.

\section{METODOS}

Entendiendo que la realidad en general y la empresarial en particular la microempresaria es una construcción compleja de significados; desde la interacción entre los individuos surgen miradas y necesidades diversas, significados particulares compartidos por los miembros de un grupo concreto, y significados que devienen y forman parte de la cultura en la cual están insertos.

En consecuencia, para esta investigación se procede desde una postura introspectiva vivencial. Desde un acercamiento sustentado en la teoría de la acción de Schön (1992), contexto en el cual los individuos de una sociedad están insertos y conformados por intereses y valores sociales, culturales, políticos y económicos en donde lo ideológico y las relaciones de poder se hacen presentes. Este contexto es el marco dentro del cual los individuos interaccionan y construyen significados.

Por todo esto, hay una preocupación por comprender el accionar desde los escenarios naturales; por lo que a través de un plano ALFA del objeto de estudio se pretende ilustrar de forma sencilla lo expuesto sobre los planos del conocimiento y la dimensión personal en la construcción de este y los niveles de desempeño laboral desplegados en la interacción con el medio. 


\section{Figura 1}

Plano ALFA del Objeto de Estudio:

\section{PLANO·ALFAR}

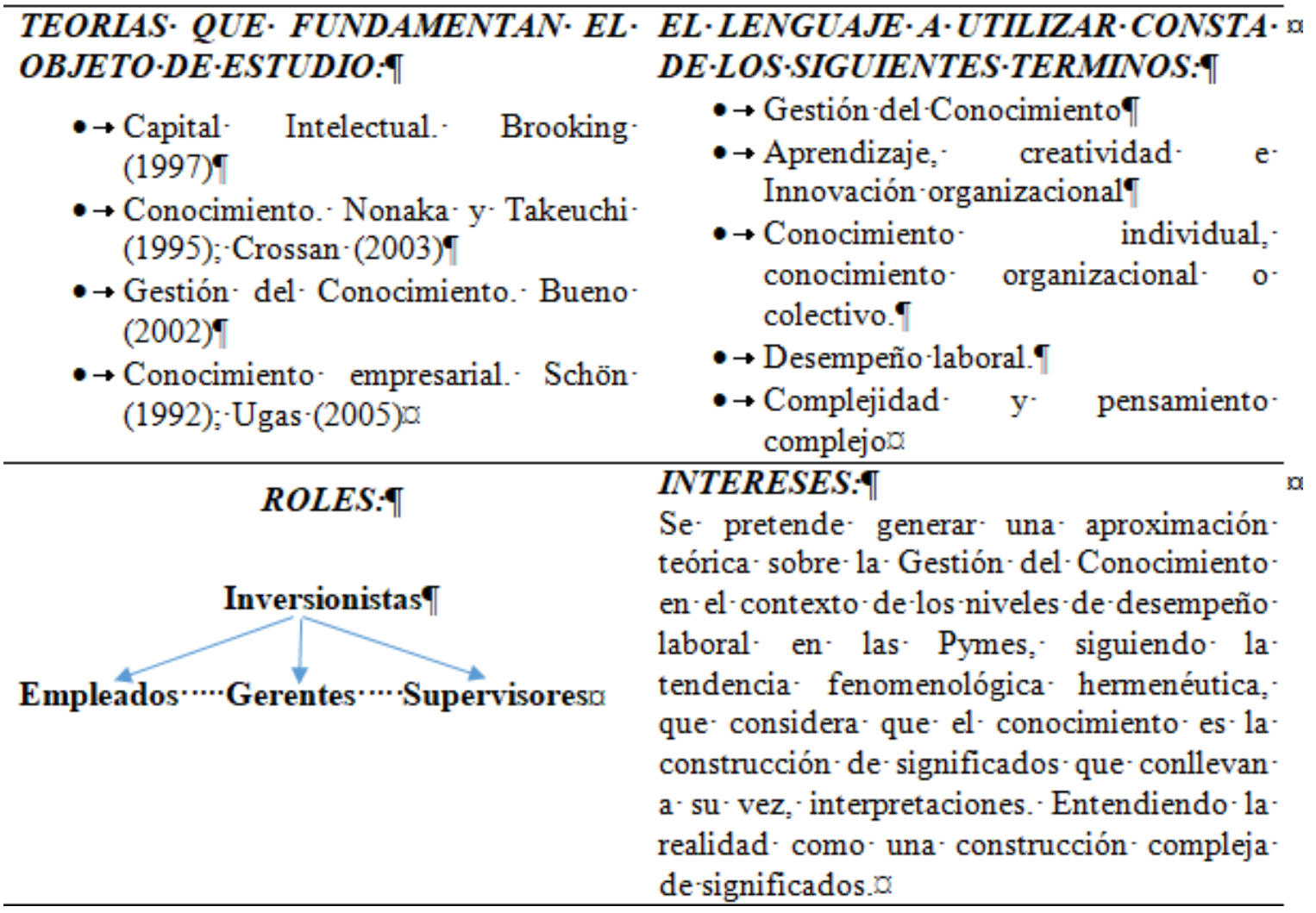

Fuente: elaboración propia (2021)

En función de la necesidad del investigador, del percibir el fenómeno a conocer, y definir como, por qué y para que se quiere conocer, conllevo a enfocar el estudio bajo un método fenomenológico-hermenéutico como fundamento teórico-operativo del desarrollo de esta investigación, considerando la pertinencia de la información que proporciona el análisis e interpretación de las instancias fenomenológicas que actúan en la manifestación de la gestión del conocimiento en contexto del desempeño laboral en las Pymes. Situándose esta investigación como un estudio Cualitativo.

Por otra parte, el abordaje fenomenológico-hermenéutico represento un gran reto para el investigador, pues de su observación y sentido dependerá gran parte de la interpretación y aproximación teórica a partir de las acciones, voces y las interacciones de los actores (empleados), por lo que debe centrarse en el cómo, qué, quiénes, integrando todos los escenarios posibles para poder conocer y construir los sentidos y significados de la gestión del conocimiento en las Pymes. En este orden, se define el carácter de este estudio, como interpretativo, concretando la manifestación del fenómeno a investigar. 
El estudio sobre la gestión del conocimiento en el contexto del nivel de desempeño laboral en las Pymes se realiza desde una postura interpretativa, ya que se busca comprender los modos específicos en los que los significados de la organización social y de la cultura se relacionan con las actividades de los empleados al realizar juntos una acción de producción.

Cuando nos interesamos desde la investigación por los significados que resguardan las acciones nos acercamos a una mirada fenomenológica. En este sentido, se pretende llegar al significado de la experiencia subjetiva de las personas; en este caso la base del conocimiento es esta experiencia subjetiva inmediata de los hechos tal como se perciben (Forner y Latorre, 1996). En efecto, interesa obtener un conocimiento profundo de la naturaleza o significado de las experiencias cotidianas (Van Mannen, 2003); es decir, desde la fenomenología se profundizará en aquellos significados que llevan a la gestión del conocimiento en el contexto del desempeño laboral en las Pymes.

Como rasgo a resaltar en este fundamento metodológico, es el sentido histórico del contexto, para la fenomenología según Ray (2003), el significado se puede interpretar solamente a través de la historia, entendiéndose que siempre ocurre en un contexto, donde las experiencias subjetivas están vinculadas al contexto social, político y cultural.

\section{Trabajo de Campo}

- $\quad$ Para obtener la información a interpretar, se aplicó la entrevista semiestructurada a través de un guion de interrogantes que se desprenden de los propósitos de la investigación. El dialogo hermenéutico con los integrantes (muestras) de los diferentes Roles, nos coloca en contacto con distintas perspectivas y visiones que refieren la realidad del inversionista, empleado, gerente y supervisor para evaluar los niveles de desempeño laboral desde el punto de vista de la gestión del conocimiento.

- Se seleccionaron informantes claves, tal como plantea Ovalles, M. (1999), constituyendo está, una técnica que todo investigador debe realizar, para asegurar la obtención pertinente de la información que se necesita, resaltando este autor, que los informantes claves se deben seleccionar intencionalmente y con base en:

○ Los propósitos de la investigación

○ La experiencia y competencia personal.

- En la investigación cualitativa existen diferentes técnicas de obtener información, seleccionando para este estudio, las siguientes: observación participativa y nota de campo; la entrevista con informantes claves; las grabaciones sonoras; el video y la fotografía, entre otros, según Martínez (2005). 


\section{E-IDEA}

J OURNAL OF BUSINESS SCIENCES

- $\quad$ A fin de interpretar y validar la información obtenida en atención a los propósitos de la investigación, la fundamentación se basó en el método propuesto por Husserl (ob.cit.), haciendo reducción eidética, como producto de la interpretación contextual de esa información, utilizando un juego de matrices, las cuales se identificaron de la siguiente manera:

- Matriz I: asignación de Categorías de Análisis y la detección de Hallazgos relevantes.

- Matriz II: Triangulación y Contextualización de los Hallazgos Relevantes en sus respectivas Categorías y Subcategorías de Análisis; Matriz II-1: Subcategorías de Análisis (interpretación contextual de toda la información, ejercicio hermenéutico profundo y racional.

○ Matriz III: matriz sinóptica 1, Categoría de Análisis.

- En cuanto a la credibilidad de la investigación, la confianza de una investigación cualitativa está basada en lo que respecta a la información, haciendo referencia a la conciencia humana. En este orden de ideas, Pérez y Countin (2005), plantea que la confiabilidad de la investigación resulta compleja, pues tanto el proceso empleado como la naturaleza de los fundamentos requieren un proceso natural irrepetible.

En este orden de ideas, esta investigación es confiable pues se hizo una descripción detallada con una profunda interpretación del contenido que se deriva del fenómeno estudiado.

\section{RESULTADOS Y DISCUSIÓN}

Entre los objetivos básicos de este estudio, esta describir la gestión del conocimiento para elevar niveles de desempeño laboral en las PYMES en el Ecuador, para generar hallazgos que den significancia y sentido a las consideraciones emergentes.

Como tema a abordar, está la sociedad del conocimiento, y en este sentido, Barney (1991), expresa que no es una sociedad de oportunidades plenas sino de grandes desafíos, siendo uno de estos, a nivel de los actores sociales, y consiste en asegurar el acceso al conocimiento y mantenerlo a partir de la capacidad de aprendizaje constante. En consecuencia, el factor humano en las organizaciones ha adquirido gran relevancia en los últimos tiempos, ya que la clave está en el conocimiento que tienen las personas, lo cual representa la principal fuente para obtener ventajas competitivas al mejorar los niveles de desempeños laborales. A su vez Arguello, C. (2017), hace referencia de gestión del conocimiento, definiéndola como una estrategia que desarrolla capacidades para originar, almacenar, transferir, aplicar y proteger el conocimiento organizacional, con el fin de incrementar el capital intelectual de las cadenas de producción, en el marco del ciclo del conocimiento, en base a proyectos, necesidades y un ambiente innovador específico. En el

Bravo Cedeño, M. C., \& Muñoz Salgado, R. J. (2021). Gestión del Conocimiento para elevar niveles de desempeño laboral en las Pymes. E-IDEA Journal of Business Sciences, 3(9), 1-14. 
mismo orden de ideas, Arguello destaca la siguiente descripción de las PYMES, entendiéndose como el conjunto de pequeñas y medianas empresas que, de acuerdo a su volumen de ventas, capital social, cantidad de trabajadores, y su nivel de producción o activos presentan características propias de este tipo de entidades económicas. Donde a su vez, Alcívar, Alarcón y Ferrin (2020), describen la microempresa ecuatoriana y la identifican esencialmente por ser de composición familiar y orientada al comercio y la venta de servicios. Sus niveles de gestión contable, gestión de talento humano y gestión del conocimiento son bastante bajos y realizan sus actividades de forma empírica y con su conocimiento tácito. En correspondencia, Naranjo (2018) acota: "Las áreas de mercadeo, ventas y talento humano, son las más vulnerables en las microempresas, ya que son las que constituyen el eje principal y al no tener los recursos óptimos adecuados pueden llevar al negocio al declive" (pág. 81).

Al respecto, Nonaka (2003), afirma que el conocimiento en forma de actitudes, valores, patentes, capacitación, métodos de trabajo, tecnología, sistemas de información, reputación corporativa y filosófica, representan la mejor alternativa para que la organización replantee una mejor estrategia que apunte hacia la innovación continua y efectiva.

Claramente, se determina que el verdadero valor de las organizaciones radica cada vez más en los recursos intangibles y cada vez menos en los elementos tangibles; maquinaria, equipo productivo, instalaciones, todo esto se puede comprar y son fáciles de transferir; sin embargo, no existen mercados donde adquirir saberes, procedimientos, medios para mejorar el servicio prestado, que de manera intelectiva y procedimental, mejoren el desempeño individual y de la organización; en otras palabras, no se dispone de "mercados para adquirir conocimientos", que por su carácter tácito son más difíciles de reproducir.

En el mismo orden, de establecer la relación entre gestión del conocimiento y el desempeño laboral en las PYMES, según Fontalvo, Quejada y Puello (2011) afirmaron que: "La gestión del conocimiento como dinamizador de la innovación empresarial, mediante la puesta en marcha de políticas en ciencia, tecnología e innovación (CT+I), cumplen un papel fundamental en la generación de ventajas competitivas en las naciones". Siendo necesario, establecer la diferencia entre información y conocimiento. La información forma parte del conocimiento, pero no es el conocimiento. El conocimiento considera las creencias que están basadas en la información. El conocimiento surge en un contexto que involucra creencias previas, interacción con personas, juicios, conductas y actitudes individuales y grupales. La información no es activa por definición, en cambio el conocimiento es activo y se asocia generalmente a individuos o grupos de individuos (Rodríguez, 2007).

De todo lo anteriormente descrito, derivan varias definiciones de términos muy importantes para llevar a cabo esta investigación, como lo son:

\section{Gestión del Conocimiento desde diversos puntos de vista documental}

Nonaka y Takeuchi (1999) esbozan “el conocimiento como la unidad analítica básica para explicar el comportamiento de las empresas” (pág. 59). 
El conocimiento es la suma de la experiencia y valores de los miembros de una organización y de la información contenida en variados sistemas y datos facilitados a estos miembros por lo tanto es intrínseco a ellos (Davenport, 1998 citado por Flores, 2010).

Sobre la definición de la gestión del conocimiento, Valhondo (2003) señala que es la transformación del conocimiento en negocios aprendiendo mediante la trasformación de información en conocimiento. Por otra parte, según Artiles (2002), es un proceso sistémico de planificar, organizar, optimizar y controlar los conocimientos en una organización, con el objetivo de desarrollar las capacidades reales y potenciales del capital intelectual que facilite su crecimiento científico, tecnológico e innovador, dando respuesta a la solución de problemas.

Ahora bien, de todos estos planteamientos se deduce, según Alcívar, Alarcón y Ferrin (2020), que la gestión del conocimiento de manera general implica integrar un complejo rango de actividades que abarca, desde la creación o captación, estructuración, transformación y transferencia de conocimiento, hasta su almacenamiento e incorporación a todos los procesos de la organización. Este conjunto de procesos permite que el capital intelectual de una institución aumente de forma significativa.

\section{Desempeño Laboral}

Según Sy Corvo, H. (2018) Es la evaluación que determina si una persona realiza bien su trabajo. Es estudiado académicamente como parte de la psicología industrial y organizacional, formando también parte de la gestión de recursos humanos.Es una evaluación a nivel individual, una medida basada en el esfuerzo de una sola persona. Generalmente el departamento de recursos humanos administrará la evaluación, pero el desempeño laboral es un proceso sumamente importante para el éxito de toda compañía.

La definición de desempeño laboral puede parecer sencilla a primera vista: se trata de qué tan bien o mal hacen los empleados su trabajo. Pero cuando se considera el impacto que este concepto tiene en el negocio, es esencial tener una mirada más profunda. Por otro lado, el desempeño ejemplar de los empleados puede aumentar la motivación y el resultado final; el departamento de recursos humanos y los supervisores individualmente deben medir regularmente el desempeño laboral de los empleados.

Según Chiavenato (2017), describe el desempeño laboral como la eficacia que tienen los empleados en los trabajos establecidos dentro de la organización y a los logros alcanzados, este desempeño varía de una persona a otra.

En este orden de definiciones Alegre y Lapiedra (2005) llegaron a la conclusión de que una vez estudiada un conjunto razonablemente homogéneo de empresas y habertrabajado con una muestra tan restringida pudo limitar la generalización de los resultados de su investigación; sin embargo, también los dotan de mayor consistencia. Su estudio confirmó 
la utilidad del enfoque de la empresa basado en las competencias. Dado que el desempeño innovador varía entre las empresas productoras de biotecnología, tratando de explicar esta asimetría a través de las competencias componente y arquitecturales. Analizando los efectos directo e indirecto, mostraron que competencias componente tales como las prácticas de gestión del conocimiento fomentaron la ventaja competitiva en innovación, principalmente de forma indirecta a través de la creación de competencias arquitecturales. La comparación de los efectos directo e indirecto indicaron que el último es el que prevalece cuando se analizan competencias componente, competencias arquitecturales y desempeño al mismo tiempo. Este estudio ofreció una explicación clara del proceso de creación de competencias.Las competencias arquitecturales, por su parte, permiten que las prácticas sean más eficaces, que su implantación sea flexible y adaptable al cambio, evitando que éstas se conviertan en rigideces organizativas y asegurando un impacto positivo en el desempeño innovador. Las competencias arquitecturales constituyen el vínculo entre las prácticas operativas y la ventaja competitiva sostenible, por tanto, los directores deberían tomarlas en consideración a la hora de formular la estrategia de la empresa.

\section{Las PYMES}

La conceptualización y las características de la microempresa o PYMES, es que han sido una organización determinante en la economía de muchos países. Existen una variedad de definiciones acerca de ellas, por lo que se escogió la del Banco Interamericano de Desarrollo (BID) 2005 que expresa que son empresas de pequeña escala, que no separan claramente las cuentas del negocio y de la familia y, en general, que operan en condiciones de alta informalidad, pueden incluir amplias categorías desde puestos de venta callejeros, panaderías, sastrerías hasta pequeños talleres para la reparación de vehículos. De acuerdo al Proyecto SALTO/USAID (2005) para Ecuador: Una microempresa es un negocio personal o familiar que emplea hasta 10 personas, el cual es poseído y operado por una persona individual, una familia, o un grupo de personas individuales de ingresos relativamente bajos, cuyo propietario ejerce un criterio independiente sobre productos, mercados y precios, además constituye una importante (si no la más importante) fuente de ingresos para el hogar. No se incluyen profesionales, técnicos medios, científicos, intelectuales, ni peones, ni jornaleros.

Una vez obtenida la información en el llenado de las matrices, es relevante indicar:

- Partiendo de la información obtenida de la Matriz I, aplicada para cada informante clave, el investigador desde la intersubjetividad reconstruye la realidad que viven, crean y recrean los sujetos o roles involucrados, para de esta manera develar el fenómeno gestión del conocimiento y los niveles del desempeño laboral en las Pymes, develando luego los ejes rectores, donde se plasmaran los hallazgos más relevantes.

Resultando: como primer eje rector, los pensamientos, sentimientos y acciones de los actores sociales (Roles), generándolos a través de sus discursos, lo cual los reviste de todo lo humano y de la humanidad que como cualidad investigativa nos lleva a dar explicaciones, argumentaciones, pero también alternativas para el mejoramiento continuo. 


\section{E-IDEA}

J OURNAL OF B USINESS SCIENCES

- Los involucrados en la información obtenida de la Matriz II, expresan al unísono, que la gerencia que ellos vivencian debe deslastrarse de los esquemas obsoletos, personalistas y amiguismos. Donde, según el punto de vista de Mora (2007), la gerencia constituye el soporte estructural de la gestión que afecta a los más variados sectores de la organización, por tanto, esta gerencia debe ser dinámica.

- La matriz III, arroja la información que permite reconocer que deben ser abordados en el proceso de transformación desde una visión gerencial como el dar la cara a las demandas sociales con respecto a la formación del talento humano. Creando valor agregado para el futuro inmediato a través de la gestión del conocimiento como elementos claves para la transformación y el desempeño laboral.

- De acuerdo a lo planteado por Pérez y Countin (2005), mostrando que la productividad es el reflejo del conocimiento y Blanco (2003), centra que lo importante no es el modelo de gestión del conocimiento sino el empoderamiento que se haga en contexto de él; así mismo se observa de la información obtenida de las matrices II y III, un alejamiento por parte de los sujetos sociales (empleados) en la configuración de un concepto relación Inversionista-empleado-gerentesupervisor.

De tal modo, que la consideración emergente es promover espacios de disertación, estudio, construcción y reflexión sobre los niveles de desempeño laboral en la gestión del conocimiento basado en el aprendizaje experiencial como una vía de revisión constante de las necesidades humanas, mostradas en la investigación temática de Freire (1998), como elemento vinculante entre los inversionistas-gerentes-empleado-supervisor.

- Algunos resultados reflejan expresiones de los sujetos claves indagados, que conceptualmente No perciben a la gestión del conocimiento como una vía para incrementar los niveles de desempeño laboral. No obstante, establecen relación gestión y gerencia dando énfasis en lo negativo, lo cual permite plantear un escenario de desarrollo personal para aperturar perspectivas en cada uno y luego abordar la gestión del conocimiento como un factor humano y humanizante para las Pymes.

- Las abstracciones y el lenguaje implícito cobra mayor énfasis en las acciones, se evidencia que el actuar lo colocan fuera de sus espacios personales y de ello depende el dinamismo que se impregne a su desempeño laboral como sujeto orgánico y colectivo.

- La turbulencia que se evidencia en las expresiones permite crear un espacio borroso en la actualidad, comprensible desde el hecho humano la incertidumbre, la complejidad organizativa, entre otros, pero da un salto cuántico cuando vemos sus expresiones hacia el futuro.

- Los hallazgos relevantes reflejan argumentos para pensar significativamente positivo en la acogida que tendría un programa de formación del talento humano para mejorar y hacer más eficientes los niveles de desempeño laboral en las Pymes. Indicando la apreciación que en el imaginario gerencial microempresarial se clama por entrar en un proceso de transformación.

Bravo Cedeño, M. C., \& Muñoz Salgado, R. J. (2021). Gestión del Conocimiento para elevar niveles de desempeño laboral en las Pymes. E-IDEA Journal of Business Sciences, 3(9), 1-14. 
- Por último y no menos importante, basados en lo que establece Urquiola, 0. (2009), si el entorno evoluciona en la dinámica de lo creativo, empoderamiento, gerenciar el conocimiento e investigar, y a los cuales se debe dar mayor énfasis, estos son tanto la formación como la capacitación, y el otro que debe subyacer en el primero es enseñar por competencias sociales, productivas, para la vida, cognitivas, emocionales y espirituales, de manera de influenciar en los niveles de desempeño laboral en sus entornos. 


\section{E-IDEA}

JOURNAL OF B USINESS SCIENCES

\section{REFERENCIAS BIBLIOGRÁFICAS}

Alegre, J. y Lapiedra, R. (2005). Gestión del conocimiento y desempeño innovador: un estudio del papel mediador del repertorio de competencias distintivas Cuadernos de Economía y Dirección de la Empresa, núm. 23, 2005, pp. 117-138 Asociación Científica de Economía y Dirección de Empresas Madrid, España.

Alcívar Mero, M.J., Alarcón Chávez,C.R., \&FerrínSchettinni, H.M.(2020). Talento humano y la gestión del conocimiento en las microempresas. Podium, 37, 71-88. doi:10.31095/podium.2020.37.6

Arguello, C. (2017). Desarrollo de un modelo de gestión del conocimiento para las PYMES del sector textil de la ciudad de Riobamba.Facultad de Ingeniería Industrial -

UNMSM. Revista Industrial Data 20(2): 79-86 (2017)DOI: http://dx.doi.org/10.15381/idata.v20i2.13944

Artiles, S. (2002). Las redes del conocimiento como producto de la gerencia de la información en ambientes. Gestión del conocimiento. Madrid: Editorial Académica.

Freire, P. (1998). Investigación temática. Brasil: Dos Santos.

Fontalvo, T., Quejada, R., \& Puello, J. (2011). La gestión del conocimiento y los procesos de mejoramiento. Dimens Empres, 9 (1), 80-87.

Forner, A. y Latorre, A. (1996). Diccionario terminológico de investigación educativa y psicopedagógica. Barcelona: EUB. 
Jaime Carriel, E. E. (2017). El Emprendimiento en Ecuador. Vision y Perspectivas. Samborondón, Ecuador: Universidad ECOTEC.

Lacalle, M. (2010). Glosario básico sobre Microfinanzas. Madrid: Foro NantikLum de MicroFinanzas.

Martínez, C.E (2005) Teoría avanzada de organización y gestión. Capítulo 4 p 118-192 Unibiblios. Colombia. Recuperado de: www.cid.unal.edu.co/files/publications/CID20050404mateav.pdf

Martinez R., Navas Wy Abad G. (2020). El desarrollo del emprendimiento a través del apoyo de políticas nacionales e internacionales. Memorias II Congreso Internacional de Fiscalidad y Finanzas ISBN: 978-9942-805-16-4

Naranjo, M. (2018). Análisis de la gestión del talento humano, mercadeo y ventas en las microempresas del cantón Milagro y su incidencia en su crecimiento y desarrollo comercial. [Tesis de Maestría]. UNEMI.

Nonaka, I., y Takeuchi, H. (1999). La organización creadora del conocimiento: Cómo las compañías japonesas crean la dinámica de la innovación. México: Oxford UniversityPress.

Ovalles, M. (1999). La investigación cualitativa, UNAM, Distrito Federal, Mexico.

Organización Mundial de la Salud (2020). La OMS caracteriza a COVID-19 como una pandemia. https://www.paho.org/es/noticias/11-3-2020-oms-caracteriza-covid19- como-pandemia

Perez\&Coutin (2005). Administración del conocimiento. Editorial Panapo. Montevideo 


\section{E-IDEA}

J OURNAL OF BUSINESS SCIENCES

Ray, M. (2003). La riqueza de la fenomenología: preocupaciones filosóficas teóricas y metodológicas. En J Morse (Ed.). Asuntos críticos en los métodos de investigación cualitativa, (pp. 139-157). Antioquia: Editorial Universidad de Antioquia.

Rodríguez Ramírez, A. (2009). Nuevas perspectivas para entender el emprendimiento empresarial. Pensamiento \& gestión, (26), 94-119.

Rodríguez, E. (2007). Gestión del conocimiento y eficacia de las organizaciones: un estudio empírico en instituciones públicas. Interciencia, 32(12), 820-826.

Schön, D. (1992). La formación de profesionales reflexivos. Hacia un nuevo diseño del aprendizaje y las profesiones. Barcelona: Paidós.

Sy Corvo, H. (2018) Desempeño laboral: características y ejemplos.

Urquiola, O. (2009). La gestión de cambio organizacional: Perfeccionamiento empresarial: Biblioteca virtual de las ciencias en Cuba. Redicen. Red Cubana de la Ciencia. \{Documento en línea\}. Disponible: http://www.blibliotecas.cu/

Valhondo, D. (2003). Gestión del conocimiento: Del mito a la realidad. Madrid: Ediciones Díaz de Santos.

Van Mannen, J. (1988). Tales of thefield. On writing ethnography. Chicago: University of Chicago Press. Vassiliadis (2000). Competing whit intelectual capital: Theoretical background.Institute for information Management and institute of management, University of St. Gallen. 